\title{
Area level fusion of Multi-focused Images using Multi-Stationary Wavelet Packet Transform
}

\author{
K. Kannan \\ Professor \& Head/ECE \\ Kamaraj College of Engineering \& \\ Technology, Virudhunagar, India
}

\author{
S. Arumuga Perumal \\ Professor \& Head \\ Department of Computer Science \\ S. T. Hindu College, Nagarcoil, India
}

\author{
K. Arulmozhi \\ Principal, Kamaraj College of \\ Engineering \& Technology, SPGC \\ Nagar, Virudhunagar, India
}

\begin{abstract}
Image fusion is defined as the process of combining two or more different images into a new single image retaining important features from each image with extended information content. There are two approaches to image fusion, namely Spatial Fusion and Transform fusion. In Spatial fusion, the pixel values from the source images are directly summed up and taken average to form the pixel of the composite image at that location. Transform fusion uses transform for representing the source images at multi scale. The most common widely used transform for image fusion at multi scale is Wavelet Transform since it minimizes structural distortions. But, wavelet transform suffers from lack of shift invariance \& poor directionality and Stationary Wavelet Transform and Wavelet Packet Transform overcome these disadvantages. The Multi-Wavelet Transform of image signals produces a non-redundant image representation, which provides better spatial and spectral localization of image formation than discrete wavelet transform. In this paper, Multi-Wavelet Transform, Stationary Wavelet Transform and Wavelet Packet Transform were combined to form Multi-Stationary Wavelet Packet Transform and its performance in fusion of multi-focused images in terms of Peak Signal to Noise Ratio, Root Mean Square Error, Quality Index and Normalized Weighted Performance Metric is presented.
\end{abstract}

\section{Keywords}

Image Fusion, Multi Wavelets, Stationary Wavelets, Wavelet Packets, Peak Signal to Noise ratio, Root Mean Square Error, Quality Index and Normalized Weighted Performance Metric.

\section{INTRODUCTION}

The multi-sensor data in the field of remote sensing, medical imaging and machine vision may have multiple images of the same scene providing different information. In machine vision, due to the limited depth-of-focus of optical lenses in Charge Coupled Devices, it is not possible to have a single image that contains all the information of objects in the image. To achieve this, image fusion is required. Image fusion is defined as the process of combining two or more different images into a new single image retaining important features from each image with extended information content. For example, Infrared and visible images may be fused as an aid to pilots landing in poor weather or Computer Tomography and Magnetic Resonance Images may be fused as an aid to medical diagnosis or millimeter wave and visual images may be fused for concealed weapon detection or thermal and visual images may be fused for night vision applications [2]. In remote sensing, the color information is provided by three sensors covering the red, green and blue spectral wavelengths. These sensors have a low number of pixels (low spatial resolution) and the small objects and details (cars, small lines, etc.) are hidden. Such small objects and details can be observed with a different sensor (panchromatic), which have a high number of pixels (high spatial resolution) but without color information. With a fusion process a unique image can be achieved containing both: high spatial resolution and color information [17]. There are two approaches to image fusion, namely Spatial Fusion (SF) and Transform fusion (TF). In Spatial fusion, the pixel values from the source images are summed up and taken average to form the pixel of the composite image at that location [15]. Image fusion methods based on Multiscale Transforms (MST) are a popular choice in recent research[16]. MST fusion uses Pyramid Transform (PT) or Discrete Wavelet Transform (DWT) for representing the source image at multi scale. PT methods construct a fused pyramid representation from the pyramid representations of the original images. The fused image is then obtained by taking an inverse PT [18]. Due to the disadvantages of PT, which include blocking effects and lack of flexibility, approaches based on DWT have begun [16]. In [16], DWT approach is considered and it uses area level maximum selection rule and a consistency verification step. But, DWT suffers from lack of shift invariance and poor directionality. One way to avoid these disadvantages is to use Dual Tree Complex Wavelet Transform (DTCWT), which is most expensive, computationally intensive, and approximately shift invariant [6-13]. But, the un-decimated DWT, namely Stationary Wavelet Transform (SWT) is shift invariant and Wavelet Packet Transform (WPT) provides more directionality. This benefit comes from the ability of the WPT to better represent high frequency content and high frequency oscillating signals in particular. The Multi Wavelet Transform (MWT) of image signals produces a non-redundant image representation, which provides better spatial and spectral localization of image formation than DWT. This paper presents the performance of Multi-Stationary Wavelet Packet Transform in multi-focused image fusion in terms of Peak Signal to Noise Ratio (PSNR), Root Mean Square Error (RMSE), Quality Index (QI) and Normalized Weighted Performance Metric (NWPM).

Section 2 of this paper describes the wavelet transform theory whereas Section 3, 4, 5, 6 presents Discrete Wavelet Transform, Stationary Wavelet Transform, Multi Wavelet Transform and Wavelet Packet Transform respectively. The Wavelet Based Image fusion and Area level Image fusion are described in Section 7 and 8. Section 9 presents the various evaluation 
measures used in this paper and Section 10 describes the experimental work.

\section{WAVELET TRANSFORM THEORY}

Wavelet theory and wavelet analysis is a relatively recent branch of mathematics. Alfred Haar developed the first wavelet in 1909. The Haar wavelet belongs to the group of wavelets known as Daubechies wavelets, which are named after Ingrid Daubechies, who proved the existence of wavelet families whose scaling functions have certain useful properties, namely compact support over an interval, at least one nonvanishing moment, and orthogonal translates. Because of its simplicity, the Haar wavelet is useful for illustrating the basic concepts of wavelet theory but has limited utility in applications. The wavelet function $\psi(\mathrm{x})$ and scaling function $\phi(\mathrm{x})$ of Haar wavelet is presented in figure1.

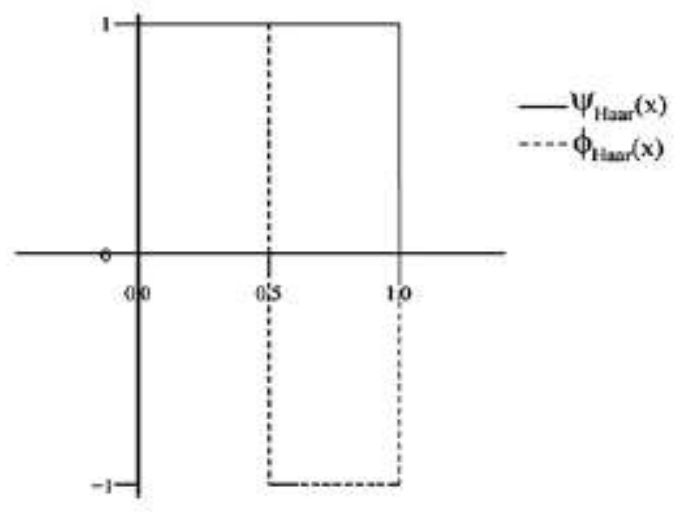

Figure 1. The wavelet and scaling function of Haar wavelet

Various researchers further developed the concept of wavelets over the next half century but it was not until the 1980's that the relationships between quadrature mirror filters, pyramid algorithms, and orthonormal wavelet bases were discovered, allowing wavelets to be applied in signal processing. Over the past decade, there has been an increasing amount of research into the applications of wavelet transforms to remote sensing, particularly in image fusion. It has been found that wavelets can be used to extract detail information from one image and inject it into another, since this information is contained in high frequencies and wavelets can be used to select a set of frequencies in both time and space. The resulting merged image, which can in fact be a combination of any number of images, contains the best characteristics of all the original images.

Wavelets can be described in terms of two groups of functions: wavelet functions and scaling functions. It is also common to refer to them as families: the wavelet function is the "mother" wavelet, the scaling function is the "father" wavelet, and transformations of the parent wavelets are "daughter" and "son" wavelets. Generally, a wavelet family is described in terms of its mother wavelet, denoted as $\psi(\mathrm{x})$. The mother wavelet must satisfy certain conditions to ensure that its wavelet transform is stably invertible[1]. These conditions are:

$$
\begin{aligned}
& \int|\Psi(x)|^{2} d x=1 \\
& \int|\Psi(x)| d x<\infty \\
& \int \Psi(x) d x=0
\end{aligned}
$$

The conditions specify that the function must be an element of $\mathrm{L}^{2}(\mathrm{R})$, and in fact must have normalized energy, that it must be an element of $L^{1}(R)$, and that it have zero mean. The third condition allows the addition of wavelet coefficients without changing the total flux of the signal. Other conditions might be specified according to the application. For example, the wavelet function might need to be continuous, or continuously differentiable, or it might need to have compact support over a specific interval, or a certain number of vanishing moments. Each of these conditions affects the results of the wavelet transform. To apply a wavelet function, it must be scaled and translated. Generally, a normalization factor is also applied so that the daughter wavelet inherits all of the properties of the mother wavelet. A daughter wavelet $\psi_{\mathrm{a}, \mathrm{b}}(\mathrm{x})$ is defined by the equation,

$$
\Psi_{a, b}(x)=a^{-1 / 2} \Psi((x-b) / a)
$$

where $\mathrm{a}, \mathrm{b} \in \mathrm{R}$ and $\mathrm{a} \neq 0$; $\mathrm{a}$ is called the scaling or dilation factor and $b$ is called the translation factor. In most practical applications it is necessary to place limits on the values of a and b. A common choice is $a=2^{-j}$ and $b=2^{-j} k$, where $j$ and $k$ are integers. The resulting equation is

$$
\Psi_{j, k}(x)=2^{1 / 2} \Psi\left(2^{j} x-k\right)
$$

This choice for dilation and translation factors is called a dyadic sampling [5]. Changing $\mathrm{j}$ by one corresponds to changing the dilation by a factor of two, and changing $\mathrm{k}$ by one corresponds to a shift of $2^{-\mathrm{j}}$. Figure 2 uses the Haar wavelet to illustrate the relationship of daughter wavelets to the mother wavelet and the effect of varying dilation and translation for both the general equation and the dyadic equation. The mother wavelet is $\psi_{1,0}(\mathrm{x})$ in Fig. 2a and $\psi^{0,0}(\mathrm{x})$ in Fig. 2b. Non-integer values are used for $\mathrm{j}$ and $\mathrm{k}$ in one example in Fig. $2 \mathrm{~b}$ to allow direct comparison with $\psi_{0.5}, 1.5(\mathrm{x})$ in Fig. $2 \mathrm{a}$. In discrete wavelet transforms, a scaling function, or father wavelet, is needed to cover the low frequencies. If the mother wavelet is regarded as a high pass filter then the father wavelet, denoted as $\varphi(x)$, should be a low pass filter. To ensure that this is the case, it cannot have any vanishing moments. It is useful to specify that, in fact, the father wavelet have a zeroth moment, or mean, equal to one:

$$
\int \phi(x) d x=1
$$

In mathematical terms, $\phi(x)$ is chosen so that the set $\{\phi(x-k)$, $\mathrm{k} \in \mathrm{Z}\}$ forms an orthonormal basis for the reference space V0. A subspace $\mathrm{Vj}$ is spanned by $\phi_{j, k}^{-}(x)=2^{1 / 2} \phi\left(2^{j} x-k\right), k \in Z$. Multiresolution analysis makes use of a closed and nested sequence of subspaces $\{\mathrm{Vj}\} \mathrm{j} \in \mathrm{Z}$, which is dense in $\mathrm{L}^{2}(\mathrm{R})$ : each subsequent subspace is at a higher resolution and contains all the subspaces at lower resolutions. Since the father wavelet is in V0, it, as well as the mother wavelet, can be expressed as linear combinations of the basis functions for $\mathrm{V} 1, \phi_{1, \mathrm{k}}(\mathrm{X})$ : 


$$
\begin{aligned}
& \phi(x)=\sum_{k} l_{k} \phi_{i, k}(x) \\
& \psi(x)=\sum_{k} h_{k} \phi_{i, j}(x)
\end{aligned}
$$

The set $\phi_{j, k}(x)=2^{1 / 2} \phi\left(2^{j} x-k\right), k \in Z$ then forms a basis for $\mathrm{Wj}$, with $\mathrm{Wj}$ being the orthogonal complement to $\mathrm{Vj}$ and $\{\mathrm{Wj}\} \mathrm{j} \in \mathrm{Z}$ forming a basis for $\mathrm{L}^{2}(\mathrm{R})$. In practice, neither the scaling function nor the wavelet function is explicitly derived. Provided that the wavelet function has compact support, the scaling function is equivalent to a scaling filter and it is sufficient to determine the filter coefficients. The coefficients $1_{\mathrm{k}}$ in Eq. (5) form this scaling, or low-pass, filter and the coefficients $h_{k}$ in Eq. (6) form the wavelet, or high-pass, filter To ensure that a signal can be exactly reconstructed from its decomposition, the scaling coefficients and wavelet coefficients must form a quadrature mirror filter.
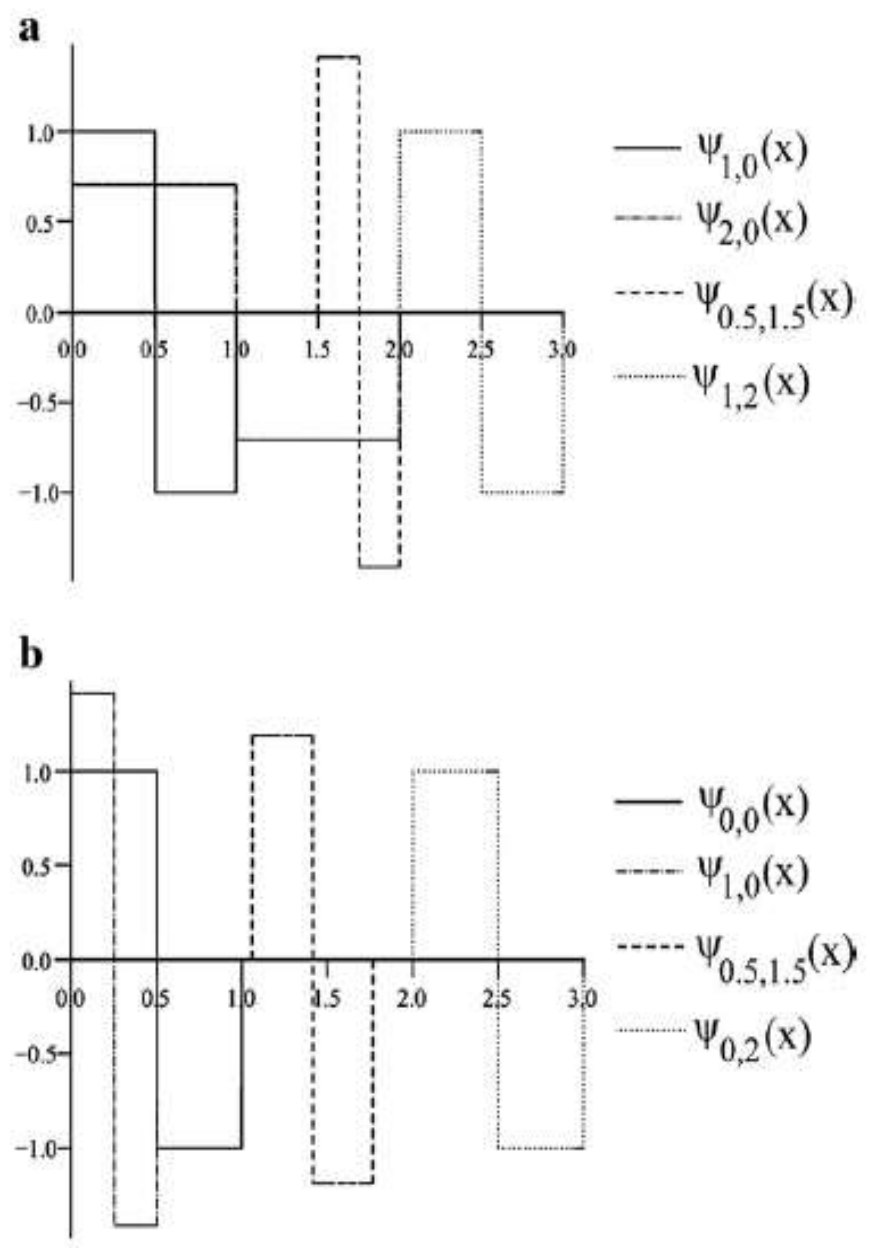

Figure 2. Mother wavelets and Daughter Wavelets
a. Daughter Wavelets according to equation 2
b. Daughter Wavelets according to equation 3

\section{DISCRETE WAVELET TRANSFORM}

Wavelet transforms provide a framework in which a signal is decomposed, with each level corresponding to a coarser resolution, or lower frequency band. There are two main groups of transforms, continuous and discrete. Discrete transforms are more commonly used and can be subdivided in various categories. Although a review of the literature produces a number of different names and approaches for wavelet transformations, most fall into one of the following three categories: decimated, un-decimated, and non-separated. A continuous wavelet transform is performed by applying an inner product to the signal and the wavelet functions. The dilation and translation factors are elements of the real line. For a particular dilation a and translation $b$, the wavelet coefficient $\mathrm{W}_{\mathrm{f}}(\mathrm{a}, \mathrm{b})$ for a signal $\mathrm{f}$ can be calculated as

$$
W_{f}(a, b)=\left\langle f, \psi_{a, b}\right\rangle=\int f(x) \psi_{a, b}(x) d x
$$

Wavelet coefficients represent the information contained in a signal at the corresponding dilation and translation. The original signal can be reconstructed by applying the inverse transform:

$$
f(x)=\frac{1}{c_{w}} \int_{-\infty}^{\infty} \int_{-\infty}^{\infty} W_{f}(a, b) \psi_{a, b}(x) d b \frac{d a}{a^{2}}
$$

where $\mathrm{C} \psi$ is the normalization factor of the mother wavelet. Although the continuous wavelet transform is simple to describe mathematically, both the signal and the wavelet function must have closed forms, making it difficult or impractical to apply. The discrete wavelet is used instead. The term discrete wavelet transform (DWT) is a general term, encompassing several different methods. It must be noted that the signal itself is continuous; discrete refers to discrete sets of dilation and translation factors and discrete sampling of the signal. For simplicity, it will be assumed that the dilation and translation factors are chosen so as to have dyadic sampling, but the concepts can be extended to other choices of factors. At a given scale $\mathbf{J}$, a finite number of translations are used in applying multi resolution analysis to obtain a finite number of scaling and wavelet coefficients. The signal can be represented in terms of these coefficients as

$$
f(x)=\sum_{k} C_{J k} \phi_{J k}(x)+\sum_{j=1}^{J} \sum_{k} d_{j k} \psi_{j k}(x)
$$

where $c_{\mathrm{Jk}}$ are the scaling coefficients and djk are the wavelet coefficients. The first term in Eq. (8) gives the low-resolution approximation of the signal while the second term gives the detailed information at resolutions from the original down to the current resolution J. The process of applying the DWT can be represented as a bank of filters, as in figure 3 . In case of a $2 \mathrm{D}$ image, a single level decomposition can be performed resulting in four different frequency bands namely LL, LH, HL and $\mathrm{HH}$ sub band and an $\mathrm{N}$ level decomposition can be performed resulting in $3 \mathrm{~N}+1$ different frequency bands and it is shown in figure 3. At each level of decomposition, the image is split into high frequency and low frequency components; the low frequency components can be further decomposed until the desired resolution is reached. In practice when wavelet decomposition is used for image fusion, one level of decomposition can be sufficient, but this depends on the ratio of the spatial resolutions 
of the images being fused. The conventional DWT can be applied using either a decimated or an un-decimated algorithm. In the decimated algorithm, the signal is down sampled after each level of transformation. In the case of a two-dimensional image, downsampling is performed by keeping one out of every two rows and columns, making the transformed image one quarter of the original size and half the original resolution. The decimated algorithm can therefore be represented visually as a pyramid, where the spatial resolution becomes coarser as the image becomes smaller. The decimated algorithm is not shift-invariant, which means that it is sensitive to shifts of the input image. The decimation process also has a negative impact on the linear continuity of spatial features that do not have a horizontal or vertical orientation. These two factors tend to introduce artifacts when the algorithm is used in applications such as image fusion.

\begin{tabular}{|c|c|c|c|}
\hline LL.3 & LH3 & \multirow{2}{*}{ LH2 } & \multirow{3}{*}{ LHI } \\
\hline Hแ. 3 & Hets & & \\
\hline \multicolumn{2}{|c|}{$\mathrm{HL} 2$} & $\mathrm{HH} 2$ & \\
\hline \multicolumn{3}{|c|}{ HL1 } & mon \\
\hline
\end{tabular}

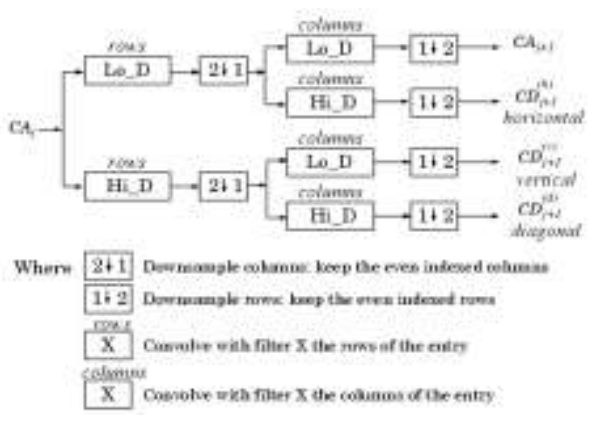

Figure 3. 2D-Discrete Wavelet Transform

\section{STATIONARY WAVELET TRANSFORM}

The Discrete Wavelet Transform is not a time- invariant transform. The way to restore the translation invariance is to average some slightly different DWT, called un-decimated DWT, to define the stationary wavelet transform (SWT). It does so by suppressing the down-sampling step of the decimated algorithm and instead up-sampling the filters by inserting zeros between the filter coefficients. Algorithms in which the filter is upsampled are called "à trous", meaning "with holes". As with the decimated algorithm, the filters are applied first to the rows and then to the columns. In this case, however, although the four images produced (one approximation and three detail images) are at half the resolution of the original, they are the same size as the original image. The approximation images from the undecimated algorithm are therefore represented as levels in a parallelepiped, with the spatial resolution becoming coarser at each higher level and the size remaining the same. The un- decimated algorithm is redundant, meaning some detail information may be retained in adjacent levels of transformation. It also requires more space to store the results of each level of transformation and, although it is shift-invariant, it does not resolve the problem of feature orientation. A previous level of approximation, resolution $\mathrm{J}-1$, can be reconstructed exactly by applying the inverse transform to all four images at resolution $\mathbf{J}$ and combining the resulting images. Essentially, the inverse transform involves the same steps as the forward transform, but they are applied in the reverse order. In the decimated case, this means up-sampling the approximation and detail images and applying reconstruction filters, which are inverses of the decomposition scaling and wavelet filters, first by columns and then by rows. For example, first the columns of the Vertical Detail image would be up-sampled and the inverse scaling filter would be applied, then the rows would be up-sampled and the inverse wavelet filter would be applied. The original image is reconstructed by applying the inverse transform to each deconstructed level in turn, starting from the level at the coarsest resolution, until the original resolution is reached. Reconstruction in the un-decimated case is similar, except that instead of up-sampling the images, the filters are down-sampled before each application of the inverse filters. Shift-invariance is necessary in order to compare and combine wavelet coefficient images. Without shift-invariance, slight shifts in the input signal will produce variations in the wavelet coefficients that might introduce artifacts in the reconstructed image. Shift-variance is caused by the decimation process, and can be resolved by using the un-decimated algorithm. Let us recall that the DWT basic computational step is a convolution followed by decimation. The decimation retains even indexed elements. But choosing odd indexed elements instead of even indexed elements could carry out the decimation. This choice concerns every step of the decomposition process, so at every level we chose odd or even.
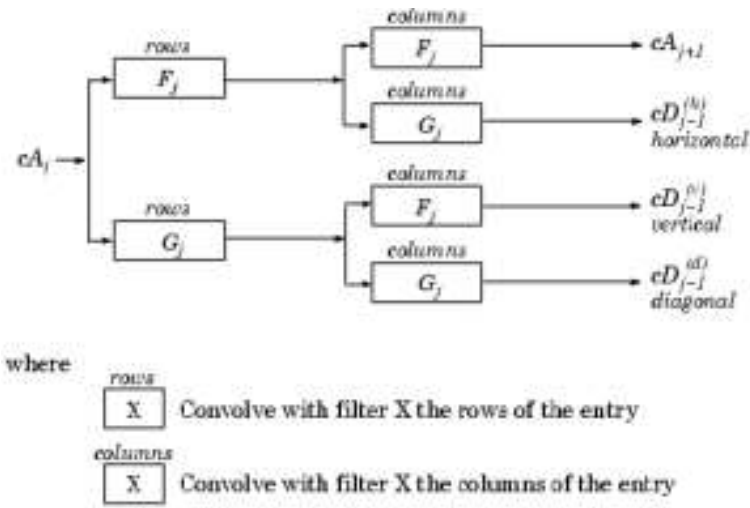

Figure 4. 2D Stationary Wavelet Transform.

If we perform all the different possible decompositions of the original signal, we have $2 \mathrm{~J}$ different decompositions, for a given maximum level $\mathrm{J}$. Let us denote by $\mathrm{j}=1$ or 0 the choice of odd or even indexed elements at step j. Every decomposition is labeled by a sequence of 0 's and 1's: = 1 , J. This transform is called the decimated DWT. It is possible to calculate all the decimated DWT for a given signal of length $\mathrm{N}$, by computing the approximation and detail coefficients for every possible sequence. The SWT algorithm is very simple and is close to the 
DWT one. More precisely, for level 1, all the decimated DWT for a given signal can be obtained by convolving the signal with the appropriate filters as in the DWT case but without down sampling. Then the approximation and detail coefficients at level 1 are both of size $\mathrm{N}$, which is the signal length. The general step $\mathrm{j}$ convolves the approximation coefficients at level $\mathrm{j}-1$, with up sampled versions of the appropriate original filters, to produce the approximation and detail coefficients at level $\mathrm{j}$. This can be visualized in the following figure 4 .

\section{MULTIWAVELET TRANSFORM}

Multiwavelets are very similar to wavelets but have some important differences. In particular, whereas wavelets have an associated scaling function $\varphi(t)$ and wavelet function $\psi(t)$, multiwavelets have two or more scaling and wavelet functions. For notational convenience, the set of scaling functions can be written using the vector notation $\varphi(t)=\left[\begin{array}{llll}\varphi_{1}(t) & \varphi_{2}(t) & \ldots & \varphi_{\mathrm{r}}(\mathrm{t})\end{array}\right]^{\mathrm{T}}$, where $\varphi(t)$ is called the multi scaling function. Likewise, the multiwavelet function is defined from the set of wavelet functions as $\psi(\mathrm{t})=\left[\begin{array}{llll}\psi_{1}(\mathrm{t}) & \psi_{2}(\mathrm{t}) & \ldots & \ldots \\ \psi_{\mathrm{r}}(\mathrm{t})\end{array}\right]^{\mathrm{T}}$. When $\mathrm{r}=1, \psi(\mathrm{t})$ is called a scalar wavelet, or simply wavelet. While in principle $r$ can be arbitrarily large, the multiwavelets studied to date are primarily for $=2$.

$$
\begin{aligned}
& \phi(t)=\sqrt{2} \sum_{k=\infty}^{-\infty} H_{k} \phi(2 t-k) \\
& \psi(t)=\sqrt{2} \sum_{k=\infty}^{-\infty} G_{k} \phi(2 t-k)
\end{aligned}
$$

However, $\left\{\mathrm{H}_{\mathrm{k}}\right\}$ and $\left\{\mathrm{G}_{\mathrm{k}}\right\}$ are matrix filters, $\mathrm{H}_{\mathrm{k}}$ and $\mathrm{G}_{\mathrm{k}}$ are $\mathrm{r} \mathrm{x} \mathrm{r}$ matrices for each integer $\mathrm{k}$. The matrix elements in these filters provide more degrees of freedom than a traditional scalar wavelet. These extra degrees of freedom can be used to incorporate useful properties into the multiwavelet filters, such as orthogonality, symmetry, and high order of approximation. The key is to figure out how to make the best use of these extra degrees of freedom. During a single level of decomposition using discrete wavelet transform, the $2 \mathrm{D}$ image data is replaced with four blocks corresponding to the sub-bands representing either low pass or high pass filtering in each direction. The multi wavelet transform have two channels, so there will be two sets of scaling coefficients and two sets of wavelet coefficients.

\begin{tabular}{|c|c||l|l|l|l|}
\hline \multirow{2}{*}{ LL } & LH & L1L1 & L1L2 & L1H1 & L1H2 \\
\hline & L2L1 & L2L2 & L2H1 & L2H2 \\
\hline & & H1L1 & H1L2 & H1H1 & H1H2 \\
\hline HL & $\mathrm{HH}$ & $\mathrm{H} 2 \mathrm{~L} 1$ & $\mathrm{H} 2 \mathrm{~L} 2$ & $\mathrm{H} 2 \mathrm{H} 1$ & $\mathrm{H} 2 \mathrm{H} 2$ \\
\hline
\end{tabular}

Figure 5. 2D Discrete and Multi Wavelet Transform

\section{DISCRETE WAVELET PACKET TRANSFORM}

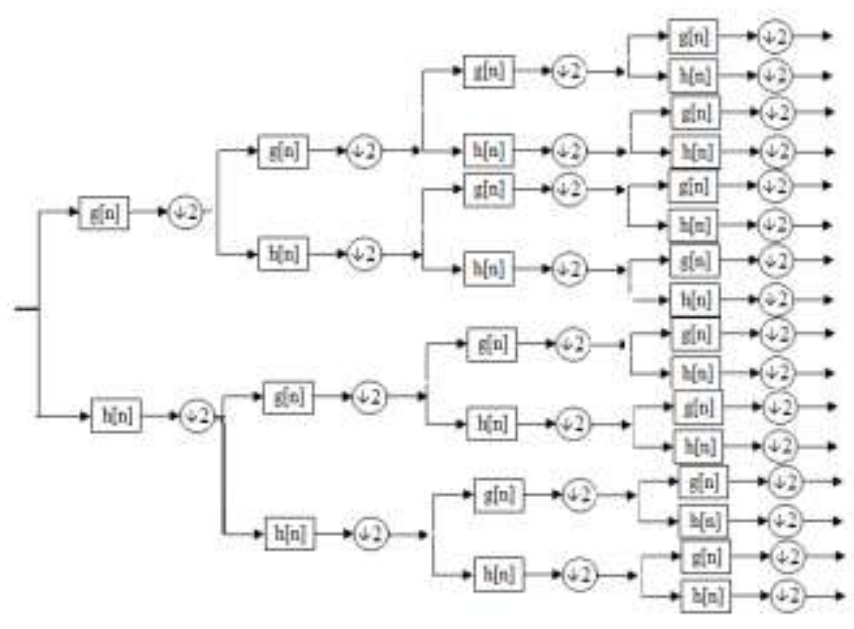

Figure 6. 2D - Wavelet Packet Transform

DWT obtained by iterating a perfect reconstruction filter bank on its low pass output, decomposes a 2D image according to octave band frequency decomposition. The DWT is far from being shift invariant and does not provide a geometrically oriented decomposition in multiple directions. For an image, the frequency decomposition provided by the DWT might not be optimal. To find a more suitable decomposition, algorithms have been proposed to find the "best-basis" from a structured dictionary of bases. For example, a best-basis algorithm that finds a sparse representation by minimizing the transformdomain entropy and an algorithm that finds the best basis in a rate-distortion sense were proposed in the literature. One way to generalize the DWT so as to generate a structured dictionary of bases is given by the discrete wavelet packet transform. The DWPT is obtained by iterating a perfect reconstruction filter bank on both its low-pass and high-pass output. However, like the DWT, the DWPT is also shift-variant and mixes perpendicular orientations in multiple dimensions. In case of a 2D image, a single level decomposition can be performed resulting in four different frequency bands namely LL, LH, HL and $\mathrm{HH}$ sub band and an $\mathrm{N}$ level decomposition can be performed resulting in $4^{\mathrm{N}}$ different frequency bands and it is shown in figure 6.

\section{WAVELET BASED IMAGE FUSION}

Wavelet transform is first performed on each source images, and then a fusion decision map is generated based on a set of fusion rules. The fused wavelet coefficient map can be constructed from the wavelet coefficients of the source images according to the fusion decision map. Finally the fused image is obtained by performing the inverse wavelet transform [2]. Let A (x, y) and B $(\mathrm{x}, \mathrm{y})$ are images to be fused, the decomposed low frequency sub images of $\mathrm{A}(\mathrm{x}, \mathrm{y})$ and $\mathrm{B}(\mathrm{x}, \mathrm{y})$ be respectively $l \mathrm{~A}_{\mathrm{J}}(\mathrm{x}, \mathrm{y})$ and $l \mathrm{~B}_{\mathrm{J}}$ $(\mathrm{x}, \mathrm{y})(\mathrm{J}$ is the parameter of resolution) and the decomposed high frequency sub images of $\mathrm{A}(\mathrm{x}, \mathrm{y})$ and $\mathrm{B}(\mathrm{x}, \mathrm{y})$ are $h \mathrm{~A}_{\mathrm{j}}^{\mathrm{k}}(\mathrm{x}, \mathrm{y})$ and $h \mathrm{~B}_{\mathrm{j}}^{\mathrm{k}}(\mathrm{x}, \mathrm{y}) .(\mathrm{j}$ is the parameter of resolution and $\mathrm{j}=1,2,3 \ldots . J$ for 
every $j, \mathrm{k}=1,2,3 .$.$) . Then, the fused high and low frequency sub-$ images $F_{j}^{k}(x, y)$ are given as $F_{j}^{k}(x, y)=A_{j}^{k}(x, y)$ if $G\left(A_{j}^{k}(x, y)\right)$ $>=\mathrm{G}\left(\mathrm{B}_{\mathrm{j}}^{\mathrm{k}}(\mathrm{x}, \mathrm{y})\right)$, else $\mathrm{F}_{\mathrm{j}}^{\mathrm{k}}(\mathrm{x}, \mathrm{y})=\mathrm{B}_{\mathrm{j}}{ }^{\mathrm{k}}(\mathrm{x}, \mathrm{y})$ and $\mathrm{F}_{\mathrm{J}}(\mathrm{x}, \mathrm{y})=l \mathrm{~A}_{\mathrm{J}}(\mathrm{x}$, $y)$ if $\mathrm{G}\left(\mathrm{A}_{\mathrm{J}}(\mathrm{x}, \mathrm{y})\right)>=\mathrm{G}\left(\mathrm{B}_{\mathrm{J}}(\mathrm{x}, \mathrm{y})\right)$, else $\mathrm{F}_{\mathrm{J}}(\mathrm{x}, \mathrm{y})=l B_{J}(\mathrm{x}, \mathrm{y})$ where $G$ is the activity measure and $F_{j}^{k}(x, y) \& F_{J}(x, y)$ are used to reconstruct the fused image $F^{\prime}(x, y)$ using the inverse wavelet transform. The block diagram representing the wavelet based image fusion is shown in figure 7.

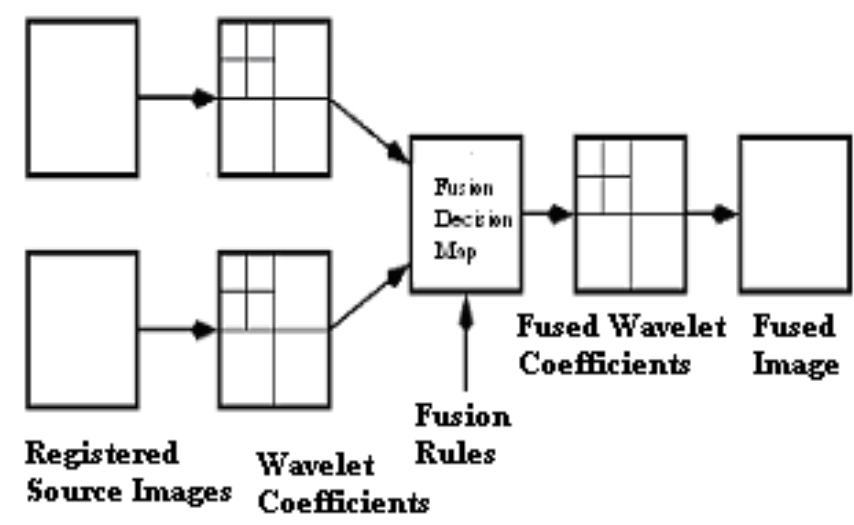

Figure7. Wavelet Based Image Fusion.

\section{AREA LEVEL IMAGE FUSION}

This section describes six methods of area level image fusion based on multi scale representation of source images using wavelets. Since the useful features in the image usually are larger than one pixel, the pixel by pixel selection rule of pixel level fusion may not be the most appropriate method. In feature level of fusion algorithm, an area based selection rule is used. The images are first decomposed into sub bands using wavelet transform. Then the feature of each image patch over $3 \mathrm{X} 3$ or $5 \times 5$ window is computed as an activity measure associated with the pixel centered in the window. To simplify the description of different feature level image fusion methods, the source images are assumed as A \& B and the fused image as F. All the methods described in this paper can be used in the case of more than two source images.

Method1: In this method, the maximum value of coefficients of sub-bands of wavelet transformed image over $3 X 3$ or $5 \times 5$ window is computed as an activity measure of pixel centered in the window. The coefficient having the larger activity measure is chosen to form the fused coefficients map. A binary decision map of same size as the wavelet transform is then created to record the selection results. This binary map is subject to consistency verification. Specifically in wavelet domain, if the centre pixel value comes from image A while the majority of the surrounding pixel values comes from image $B$, the centre pixel value should be switched to that image B. This method is called consistency verification method.

Method 2: In this method, the maximum absolute value over $3 \mathrm{X} 3$ or $5 \mathrm{X} 5$ window is computed as an activity measure of pixel centered in the window. The coefficient whose activity measure is larger was chosen to form the binary decision map and the consistency verification is applied to form the fused coefficients map [16].
Method3: This fusion scheme is the weighted average scheme suggested by Burt and Kolezynski (1993). This salient features are first identified in each source image. This salience of a feature is computed as a local energy in the neighborhood of a coefficient.

$$
\mathrm{E}(\mathrm{A}, \mathrm{p})=\sum_{\varphi=\mathrm{Q}} \mathrm{W}(\mathrm{q}) \mathrm{C}_{\mathrm{j}}^{2}(\mathrm{~A}, \mathrm{q})
$$

where $\mathrm{w}(\mathrm{q})$ is a weight and $\sum_{\varphi=\mathrm{Q}} \mathrm{w}(\mathrm{q})=1$. In practice, the neighborhood $\mathrm{Q}$ is small (typically $5 \mathrm{X} 5$ or $3 \mathrm{X} 3$ ) window centered at the current coefficient position. The closer the point $q$ is near the point $P$, the greater $w(q)$ is $E(B, p)$ can also be obtained by this rule. The selection mode is implemented as:

$$
\mathrm{Cj}(\mathrm{F}, \mathrm{p})=\left\{\begin{array}{l}
\mathrm{Cj}(\mathrm{A}, \mathrm{p}), \mathrm{E}(\mathrm{A}, \mathrm{p}) \geq \mathrm{E}(\mathrm{B}, \mathrm{p}) \\
\mathrm{Cj}(\mathrm{B}, \mathrm{p}), \mathrm{E}(\mathrm{B}, \mathrm{p})>\mathrm{E}(\mathrm{A}, \mathrm{p})
\end{array}\right\}
$$

This selection scheme helps to ensure that most of the dominant features are incorporated into the fused image.

Method 4: In this fusion method, the salience measure of each source image is computed using Equation 13. At a given resolution level $\mathrm{j}$, this fusion scheme uses two distinct modes of combination namely Selection and Averaging. In order to determine whether the selection or averaging will be used, the match measure $\mathrm{M}(\mathrm{p})$ is calculated as

$$
M(p)=\frac{2 \sum_{\varphi=Q} W(q) C_{j}(A, q) C_{j}(B, q)}{E(A, p)+E(B, p)}
$$

If $\mathrm{M}(\mathrm{p})$ is smaller than a threshold $\mathrm{T}$, then the coefficient with the largest local energy is placed in the composite transform while the coefficient with less local energy is discarded. The selection mode is implemented as

$$
\mathrm{Cj}(\mathrm{F}, \mathrm{p})=\left\{\begin{array}{l}
\mathrm{Cj}(\mathrm{A}, \mathrm{p}), \mathrm{E}(\mathrm{A}, \mathrm{p}) \geq \mathrm{E}(\mathrm{B}, \mathrm{p}) \\
\mathrm{Cj}(\mathrm{B}, \mathrm{p}), \mathrm{E}(\mathrm{B}, \mathrm{p})>\mathrm{E}(\mathrm{A}, \mathrm{p})
\end{array}\right\}
$$

Else if $M(p) \geq T$, then in the averaging mode, the combined transform coefficient is implemented as

$$
\mathrm{Cj}(\mathrm{F}, \mathrm{p})=\left\{\begin{array}{l}
\mathrm{W}_{\max } \mathrm{Cj}(\mathrm{A}, \mathrm{p})+\mathrm{W}_{\min } \mathrm{Cj}(\mathrm{B}, \mathrm{p}), \mathrm{E}(\mathrm{A}, \mathrm{p}) \geq \mathrm{E}(\mathrm{B}, \mathrm{p}) \\
\mathrm{W}_{\max } \mathrm{Cj}(\mathrm{B}, \mathrm{p})+\mathrm{W}_{\min } \mathrm{Cj}(\mathrm{A}, \mathrm{p}), \mathrm{E}(\mathrm{B}, \mathrm{p})>\mathrm{E}(\mathrm{A}, \mathrm{p})
\end{array}\right\}
$$

where

$$
\left.\mathrm{W}_{\min }=0.5-0.5\left(\frac{1-\mathrm{M}(\mathrm{p})}{1-\mathrm{T}}\right) \text { and } \mathrm{W}_{\max }=1-\mathrm{W}_{\min }\right)
$$

In this study, the fusion methods are implemented using the parameters such as a window size $3 * 3$ and a T-value of 0.75 .

Method 5: For a function $\mathrm{f}(\mathrm{x}, \mathrm{y})$ it is common practice to approximate the magnitude of the gradient by using absolute values instead of squares and square roots [14]:

$$
\nabla f=|G x|+|G y|=|f(x, y)-f(x+1, y)|+|f(x, y)-f(x, y+1)|
$$

This equation is simpler to compute and it still preserves relative changes in grey levels. In image processing, the difference between pixel and its neighbors reflect the edges of the image. Firstly compute the difference between the low frequency coefficient at the point $\mathrm{p}$ and its eight neighbors, respectively. 
The value $\mathrm{E}$ is acquired by summing squares of all the differences. At last, choose the low frequency coefficient with the greater value $\mathrm{E}$ as the corresponding coefficient of the fused image. This method can maintain the information of edges. So it can improve the quality of the fused image. The algorithm is as follows.

$$
\begin{aligned}
& E(A, p)=\sum_{\varphi=Q}\left|C_{j}(A, q)-C_{j}(A, q)\right|^{2} \\
& E(B, p)=\sum_{\varphi=Q}\left|C_{j}(B, q)-C_{j}(B, q)\right|^{2}
\end{aligned}
$$

Finally, select the corresponding high frequency coefficient of the fused image.

$$
C j(F, p)=\left\{\begin{array}{l}
C j(A, p), E(A, p) \geq E(B, p) \\
C j(B, p), E(B, p)>E(A, p)
\end{array}\right\}
$$

\section{EVALUATION CRITERIA}

There are four evaluation measures are used in this paper, namely Root Mean Square Error (RMSE), Peak Signal to Noise Ratio (PSNR), Quality Index (QI)[4] and Normalized Weighted Performance Metric (NWPM) [3] which are given in the equations $18,19,20 \& 21$ respectively.

$$
\begin{gathered}
\mathrm{RMSE}=\sqrt{\frac{\sum_{i=1}^{N} \sum_{j=1}^{N}[R(i, j)-F(i, j)]^{2}}{N^{2}}} \\
\mathrm{PSNR}=10 \log _{10}(255)^{2} /(\mathrm{RMSE})^{2}(\mathrm{db}) \\
\mathrm{QI}=\frac{4 \sigma_{a b} a b}{\left(a^{2}+b^{2}\right)\left(\sigma_{a}{ }^{2}+\sigma_{b}{ }^{2}\right)} \\
N W P M=\frac{\sum \forall i, j Q_{i j}^{A F} W_{i j}^{A}+Q_{i j}^{A F} W_{i j}^{B}}{\sum \forall i, j W_{i j}^{A}+W_{i j}^{B}}
\end{gathered}
$$

where $\mathrm{A}$ and $\mathrm{B}$ are the input images, $\mathrm{R}$ is the reference image, $\mathrm{F}$ is the fused image, $a$ is the average value of $A, b$ is the average value of $\mathrm{B}, \mathrm{Q}^{\mathrm{AF}}(\mathrm{i}, \mathrm{j})$ and $\mathrm{Q}^{\mathrm{BF}}(\mathrm{i}, \mathrm{j})$ are the edge preservation values.

\section{EXPERIMENTAL WORK}

Three pairs of source images namely Pepsi image of size $512 \times 512$, Lab and Disk image of size 480 x 640 are taken. The pairs of source images to be fused are assumed to be registered spatially. The images are wavelet transformed using Haar in the first level decomposition and sym8 for the second level of decomposition. For taking the stationary wavelet transform of the two images, readily available MATLAB routines are taken. In each sub-band, individual pixels of the two images are compared based on the fusion rule that serves as a measure of activity at that particular scale and space. Taking pixels from the wavelet transform that shows greater activity at the level creates a fused wavelet transform. The inverse wavelet transform is the fused image with clear focus on the whole image.

\section{RESULTS}

For the above mentioned method, image fusion is performed using Multi Stationary Wavelet Packet Transform (MSWPT), its performance is measured in terms of Root Mean Square Errors, Peak Signal to Noise Ratio, Quality Index \& Normalized Weighted Performance Metric and the results are shown in figure 8 and tabulated in table1.

Table 1. Performance Comparison of MSWPT

\begin{tabular}{|c|c|c|c|c|c|}
\hline \multicolumn{7}{|c|}{ Lab Image of size $480 \times 640$} \\
\hline $\begin{array}{c}\text { Metho } \\
\text { RMSE }\end{array}$ & 1 & 2 & 3 & 4 & 5 \\
\hline PSNR & 29.792 & 30.139 & 29.166 & 30.467 & 33.737 \\
\hline QI & 0.985 & 0.9862 & 0.983 & 0.9864 & 0.994 \\
\hline NWPM & 0.5849 & 0.6045 & 0.5957 & 0.6175 & 0.658 \\
\hline \multicolumn{7}{|c|}{ Disk Image of size $480 \times 640$} \\
\hline $\begin{array}{c}\text { Metho } \\
\text { d }\end{array}$ & 1 & 2 & 3 & 4 & 5 \\
\hline RMSE & 10.732 & 10.459 & 11.674 & 10.03 & 7.619 \\
\hline PSNR & 27.517 & 27.74 & 26.783 & 28.104 & 30.492 \\
\hline QI & 0.9748 & 0.9763 & 0.9712 & 0.976 & 0.9863 \\
\hline NWPM & 0.5745 & 0.6003 & 0.5869 & 0.6066 & 0.6472 \\
\hline \multicolumn{7}{|c|}{ Pepsi Image of Size $512 \times 512$} \\
\hline $\begin{array}{c}\text { Metho } \\
\text { d }\end{array}$ & 1 & 2 & 3 & 4 & 5 \\
\hline RMSE & 6.436 & 6.103 & 7.176 & 5.287 & 4.295 \\
\hline PSNR & 31.958 & 32.419 & 31.012 & 33.666 & 35.471 \\
\hline QI & 0.9906 & 0.9917 & 0.9889 & 0.9931 & 0.9955 \\
\hline NWPM & 0.6936 & 0.7058 & 0.6993 & 0.7362 & 0.7398 \\
\hline
\end{tabular}

\section{CONCLUSION}

This paper presents the comparison of five types of area level of fusion of multi-focused images using MSWPT in terms of various performance measures. MSWPT provides very good results both quantitatively and qualitatively for area level fusion. Hence using these fusion methods, one can enhance the image with high geometric resolution. 

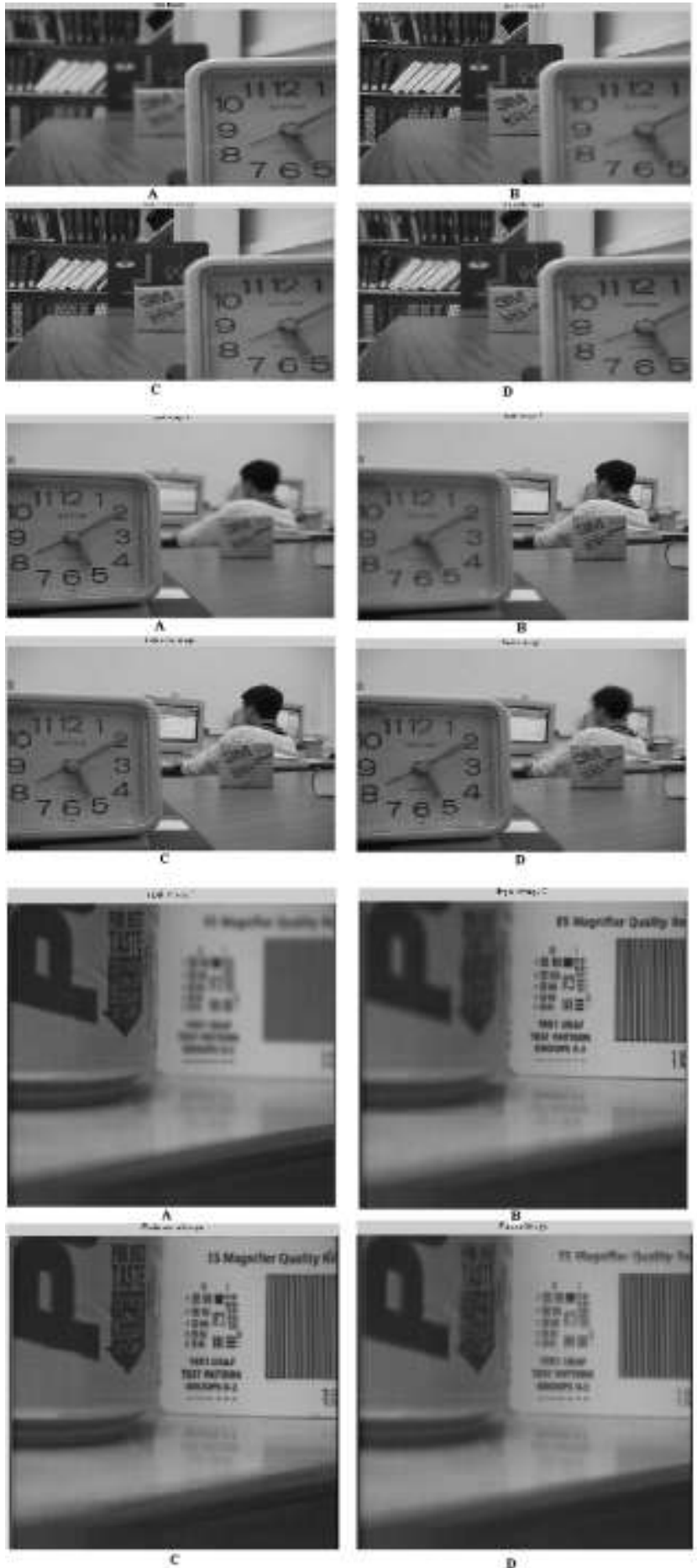

Figure 8. Results of Image Fusion

\section{A. Input Image 1 B. Input Image 2 C. Reference Image}

\section{Fused Image}

\section{REFERENCES}

S. Mallat, Wavelet Tour of Signal Processing, New York, Academic Press, 1998.
Rick S. Blum and Yang Jin zhong, 2006, Image Fusion Methods and Apparatus, US Patent, WO/2006/017233.

C.S. Xydeas and V. Petrovic, "Objective Image Fusion Performance Measure", Electronics Letter, Vol.36, N0.4, pp. 308-309, 2000.

Zhou Wang and Alan C. Bovik, "A Universal Image Quality Index”, IEEE Signal Processing Letters, Vol. 9, No.3, pp. 81-84, March, 2002.

S. Mallat, A theory for Multiresolution signal decomposition: The wavelet representation, IEEE transaction pattern anal. Machine Intell., vol. 11, no. 7, pp 674-693, July 1989.

N. G. Kingsbury, "Image processing with complex wavelets", Philos. Trans. R. Soc. London A, Math. Phys. Sci., 357(1760):2543-2560, September 1999.

N. G. Kingsbury, "Complex wavelets for shift invariant analysis and filtering of signals" Journal of Appl. and Comp. Harmonic Analysis, 10(3):234-253, May 2001.

I.W.Selesnick. "Smooth wavelet tight frames with zero moments", Journal of Appl. Comput. Harmon. Anal. 2001, 10(2), 163-181.

N.G. Kingsbury, "Complex wavelets for shift invariant analysis and filtering of signals" Applied Computational Harmonic analysis, vol. 10, no.3, pp 234-253, May 2001.

I. W. Selesnick, "The design of approximate Hilbert transform pairs of wavelet bases", IEEE Trans. Signal Processing, 50(5):1144-1152, May 2002.

I. W. Selesnick, R. G. Baraniuk, and N. G. Kingsbury, "The dual-tree complex wavelet transforms - A coherent framework for Multiscale signal and image processing" IEEE Signal Processing Magazine, 22(6):123-151, November 2005.

R. $\mathrm{Yu}$ and H. Ozkaramanli, "Hilbert transform pairs of orthogonal wavelet bases: Necessary and sufficient conditions" IEEE Trans. Signal Processing, 53(12):4723-4725, December 2005.

N.G. Kingsbury, "The dual tree complex wavelet transform: A technique for shift invariance and directional filters" in proc. of 8th IEEE DSP Workshop, Utah, paper no. 86 August 9-12, 1998.

Zhu Shu-long, Image Fusion using Wavelet Transform", Symposium on Geospatial Theory, Processing and Applications, pp. 5-9, 2004.

P. J. Burt and R. J. Kolczynski, "Enhanced image capture through image fusion", proceedings of the 4th International Conference on Computer Vision, pp. 173-182, 1993.

H. Li, B.S. Manjunath, and S.K. Mitra, "Multi-sensor image fusion using the wavelet transform", Proceedings of the conference on 'Graphical Models and Image Processing', pp. 235-245, 1995.

Gonzalo Pajares and Jesus Manuel de la Cruz, " A Wavelet based Image fusion - Tutorial", Pattern Recognition 37(2004), 1855-1872.

P.J. Burt and E. Adelson,1983, “ The Laplcian Pyramid as a Image Codec", IEEE Transactions on Communications, Vol. Com-31, No.4, pp. 532-540. 